\title{
A Comparison of Phase Unwrapping Techniques in Synthetic Aperture Radar Interferometry
}

\author{
Ana Vidal-Pantaleoni, "Rafael Oviol, Miguel Ferrando \\ Departamento de Comunicaciones, Universidad Politécnica de Valencia \\ Camino de Vera s/n, E-46071 Valencia, Spain \\ Tel.: +34-963879710, Fax: +34-963877309, E-mail: avidal@dcom.upv.es
}

\begin{abstract}
In this work, a comparison of different Phase Unwrapping techniques based on the Least Mean Square error is presented. A testing environment based on simulated interferograms has been created in order to assess the methods described in the literature. Each of them has shown good properties under different constraints. Multigrid with a previous adaptive Maximum Likelihood gradient estimation is very robust when strong aliasing is not expected. In a general scenario with aliasing, an adaptive multiresolution gradient estimator gives a coarse approximation to the low resolution topography.
\end{abstract}

\section{INTRODUCTION}

In the last years, growing potential has been identified in the field of Synthetic Aperture Radar (SAR) Interferometry. Traditionally, SAR data have been exploited in amplitude for retrieving reflectivity information. For that purpose, phase data was discarded after SAR focusing. However, interesting applications using SAR Interferometry have appeared recently. The combination of acquisitions from slightly different positions can provide accurate Digital Elevation Models (DEMs) as explained in [1]. It brings strong advantages over classical stereoscopic optical imaging like independence on natural illumination or recognizable targets. Nevertheless, it presents very difficult stages like Phase Unwrapping, which is the object of study in this paper.

Phase Unwrapping has been a subject of interest in different areas. However, it still represents a challenge for SAR applications because there are a number of conditions that complicate the process. First of all, it is a twodimensional problem. Secondly, the wrapped phase is distorted by thermal noise and severe SAR Speckle noise [1]. Besides, poor coherence caused by temporal and angular reflectivity decorrelation is usually expected. And finally, when the terrain slope is comparable to the SAR elevation angle, then undesired artifacts like shadowing, layover or foreshortening appear in the image. As a consequence of all these adverse conditions, an ideal Phase Unwrapping process should be capable to deal with noise, poor coherence, phase inconsistency and aliasing. The goal of this study is to test the different techniques and to select the potential benefits of each one in a combined environment like the one presented by Davidson and Bamler in [2].

This work has been done thanks to a collaboration scheme between Estudio Atlas S.A. and the 'Universidad Politécnica de Valencia'.

\section{PHASE UNWRAPPING}

The wrapped phase comes in the range of $[0,2 \pi]$ and the goal of Phase Unwrapping is to get a continuous phase out of it. There are different approaches to the problem, some of them described in [1,2]: region growing, path dependent methods, the recent network programming theory and others. Nevertheless, this study focuses on the application of the Least Mean Square Error approach. It provides an elegant way of formulation leading to a Partial Derivative Equation (PDE). Therefore, existing numerical methods can be used to solve the problem.

In that case, Phase Unwrapping can be divided in two stages. The first one is the estimation of the gradient of the interferogram phase. The second step is to integrate the gradient. The complexity of the overall algorithm can be higher in either of the stages, but the gradient estimation is very important, since it retains the information for the rest of the processing chain.

\section{Phase Gradient Integration through LMS}

The formulation of the LMS phase gradient integration is extensively explained in $[1,4]$. The aim of the procedure is the integration of the continuous partial derivatives. Therefore, the input to the process is an estimate of the phase. The minimization of the mean square error between the estimated phase and the true unwrapped phase leads to the resolution of the Poisson Equation. That classical equation can be solved through different techniques described in [1]. The most efficient is the direct method using Fast Fourier Transform (FFT) or Discrete Cosine Transform (DCT). In this case, no weighting can be applied in the computation of the unwrapped phase.

When taking a real interferogram, the coherence information is giving a detailed measure of phase confidence. So, a more sophisticated method taking into account a weighting function with high efficiency is introduced by [3]. In this second approach, a recursion scheme like GaussSeidel relaxation is used within a hierarchical pyramid to speed up the convergence. Gauss-Seidel is basically a filtering operation that minimizes the error between one pixel and their neighbors but it propagates very slowly the true solution throughout the global surface. The multigrid convergence to the final solution at several resolution levels is shown in Fig. 1. It provides a faster result than other algorithms like Pre-Conditioned Gradient [1] and it can 


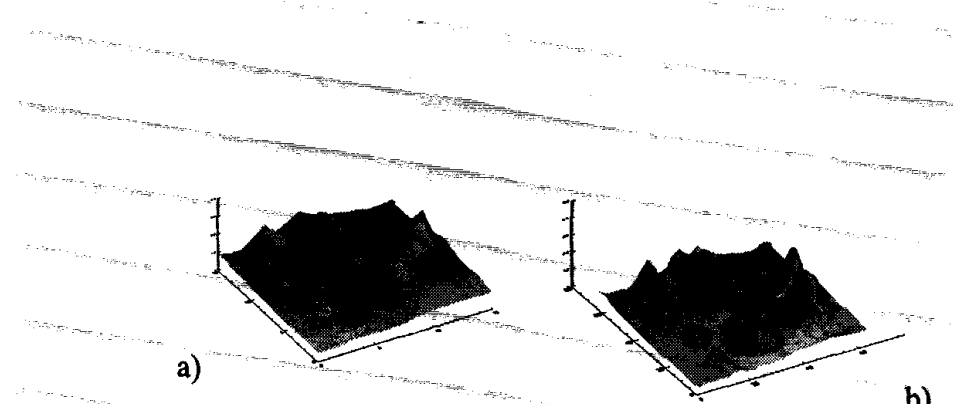

c)

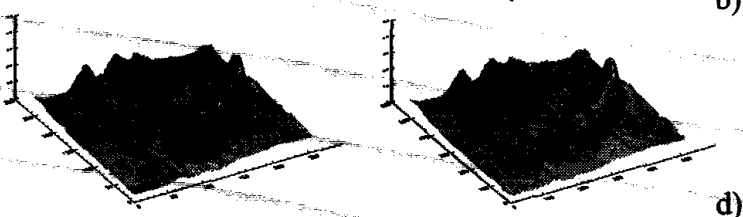

Fig. 1. Multigrid at different levels of resolution: a) $16 \times 16$, b) $64 \times 64$, c) $256 \times 256$, d) $512 \times 512$

integrate a weighting function. That fact is very important to get a correct unwrapped phase because low coherence and noise usually introduce a high amount of error in the unwrapped phase.

As a summary, multigrid is a flexible and efficient LMS estimator that can incorporate weighting values in the process. On the contrary, a direct method (FFT) should be used when a coarse model is desired as shown in [2].

\section{Phase Gradient Estimation}

Several techniques have been implemented in order to identify its main benefits and drawbacks. The first one is the most immediate: phase differences. The gradient is estimated by the phase difference between neighbor pixels. Although it is the most simple and also the less accurate method, it is a very efficient procedure that could be used when a coarse approximation of the wrapped interferogram is needed in a combined approach using coarse products like the multiresolution method with low resolution refinements introduced in [2]:

The other implemented methods for getting the partial derivatives are based on obtaining a good estimate of the Instantaneous Frequency (IF) $[1,4]$. The IF approximates properly the phase gradient under certain conditions.

One strategy is to carry out an estimate by the Maximum Likelihood (ML) method [4]. The implementation of the method consists of looking for the maximum of the spectrum of a window through a cearse search via FFTs and a fine search using Powell method. The $\mathrm{ML}$ estimate converges to the solution with a variance equal to the statistically optimum Cramer-Rao bound when the Signal to Noise Ratio (SNR) is high. However, even when it is not the case, it gives a good approximation, given that a critical threshold SNR is not reached. For that reason, an adaptive scheme that adjusts the size of the estimation window to the SNR has been also tested. The main drawback of this powerful method is that it does not cope well with aliasing conditions as pointed out in [2]. When strong aliasing is expected from topography effects or poor coherence, then a more suitable technique is provided by a multiresolution approach $\{2]$.

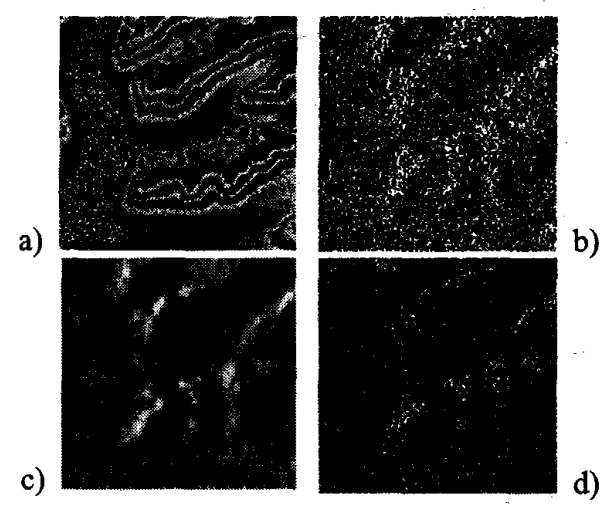

Fig. 2. IF estimators: a) Interferogram, b) Differences

c) Adaptive ML, c) Multiresolution (enhanced contrast)

Multiresolution IF estimation is given by a hierarchical decomposition with different resolution levels. Each level produces a refinement in the IF estimate as described in [2]. The problem of this method is that the interferogram noise is kept in the wrapped data. The comparison among several gradient estimation methods is shown in Fig. 2. The horizontal derivative of a simulated noisy interferogram has been analyzed with simple differences, adaptive ML and plain multiresolution. The first technique gives a poor and noisy result. Adaptive ML provides an accurate and smooth output and multiresolution gives a noisy approximation.

For obtaining a smoother result, a refinement of the multiresolution method with overlapped windows [2] can be used. In that version, no decimation is carried out in the first levels of higher resolution, only filtering is performed. By such procedure, maximum resolution is kept in those levels and a smoother result is expected. Situations with strong aliasing like in Fig. 3. can be properly unwrapped. In the example, multiresolution IF estimation is performed and compared to the simple difference estimate over a noisy Gaussian surface whose interferogram shows a dense pattern of fringes. The simple difference scheme fails to compute the

a)

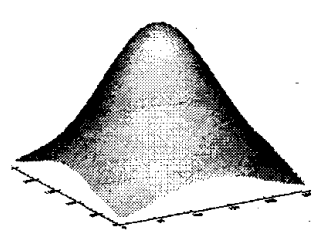

c)
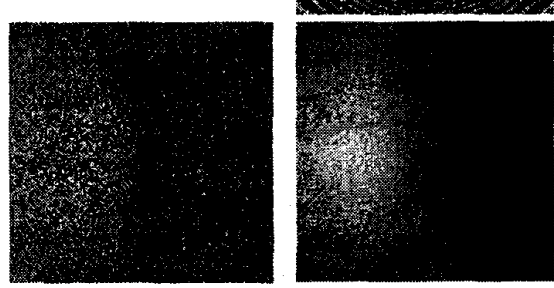

d)

Fig. 3. IF estimation: a) Gaussian surface, b) Noisy interferogram, c) Simple differences, d) Multiresolution 


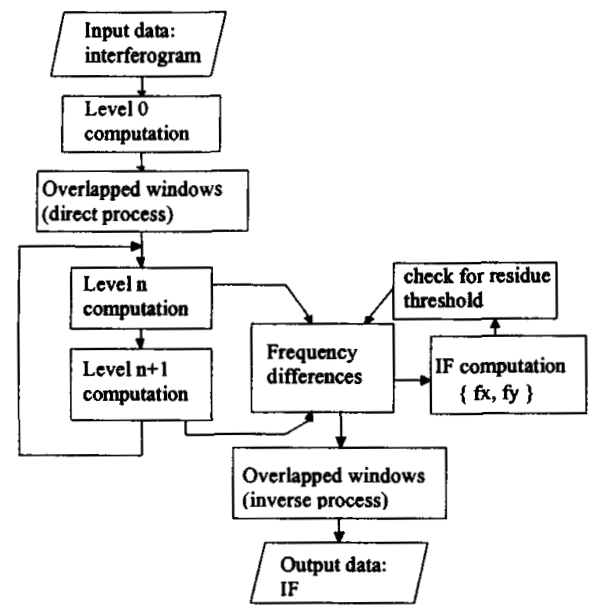

Fig. 4. Adaptive multiresolution IF estimator

horizontal partial derivative, showing a high amount of phase inconsistency.

For cases with severe aliasing, an adaptive multiresolution process described is introduced in [2] and shown in Fig. 3. In this version, the residues of the gradient field are computed at each resolution. For that purpose, the IF values are obtained at each level and if the residue magnitude exceeds a certain threshold, no information is propagated through the pyramid as described in [2]. By this procedure, the size of the real estimation window is adapted dynamically to the interferogram quality in a similar way to the adaptive $\mathrm{ML}$ method. The multigrid LMS strategy also takes advantage of a multiresolution decomposition but the goal in that case is to speed up the Gauss-Seidel relaxation scheme.

A simulation was carried out in order to assess the ability of the adaptive multiresolution approach to deal with aliasing effects. The test area is a rough terrain and its corresponding interferogram contains a pattern of fringes with a wide range of gradients. A method based on the simple differences or adaptive $\mathrm{ML}$ would fail in such scenario. On the contrary, an adaptive multiresolution IF estimator combined with a LMS gradient integration as described in [2] and shown in Fig. 4. copes with such circumstance. The noisy interferogram with aliasing and the true DEM are shown in Fig. 5. and the multiresolution reconstruction and the absolute error are shown in Fig. 6. It can be seen that the aliasing has not affected the unwrapping process because the absolute error is smaller than one full phase cycle.

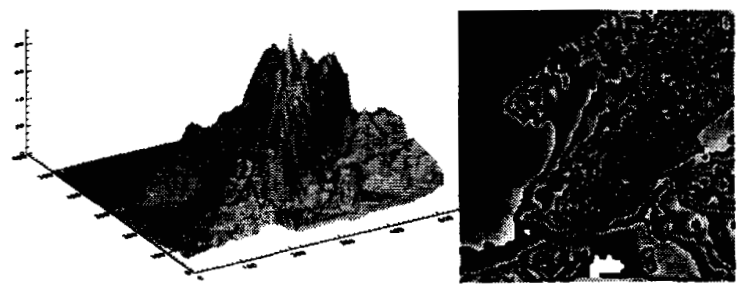

Fig. 5. Original DEM and interferogram

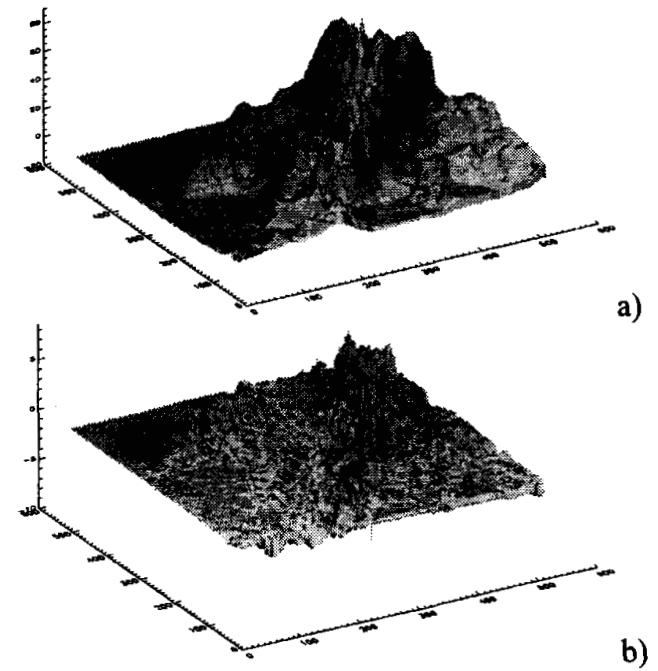

Fig. 6. Adaptive multiresolution:

a) Reconstruction, b) Absolute error $(<2 \pi)$

\section{DISCUSSION}

A number of different techniques recently appeared in the literature have been implemented and analyzed. The problem of Phase Unwrapping presents several challenges depending on the data. Each procedure has been proven to be the best under certain assumptions. Some of those are: presence of aliasing, shadowing and layover, low coherence, high amount of noise or high efficiency requirements. With regard to the LMS algorithm, FFT is adequate for coarse approximations and multigrid is more interesting for a finer result. Concerning IF estimation, adaptive ML gives very accurate results when no severe aliasing is expected and adaptive multiresolution is more robust and noisy. As a logical consequence, the optimum selection would combine both techniques following the approach pointed out in [2]. Obviously, interferogram generation using multiple data sources like different frequency bands or low resolution stereoscopic DEMs could refine even more the final result.

\section{REFERENCES}

[1] D. C. Ghiglia, and M. D. Pritt, Two-Dimensional Phase Unwrapping: Theory, Algorithms and Software. Wiley: New York, 1998.

[2] G. W. Davidson and R. Bamler, "Robust 2-D Phase Unwrapping based on multiresolution". In SPIE Proc. vol. 2958, pp. 227-237, 1996.

[3] M. D. Pritt, "Phase Unwrapping by means of multigrid techniques for Interferometric SAR", IEEE Trans. Geo Remote Sensing, vol. 34, no. 3, pp. 728-738. 1996.

[4] U. Spagnolini, "2-D Phase unwrapping and instantaneous frequency estimation". IEEE Trans. Geosci. Remote Sensing, vol. 33, no. 3, May 1995. 